\title{
Outermost tensile strain dominated exciton emission in bending $\mathrm{CdSe}$ nanowires
}

\author{
Xuewen Fu' ${ }^{1}$, Zhi-Min Liao ${ }^{1,2 *}$, Yu Ye ${ }^{1}$, Jun $\mathrm{Xu}^{1}$, Lun Dai ${ }^{1,2}$, Rui Zhu ${ }^{1}$, Wanlin Guo ${ }^{3}$ and Dapeng $\mathrm{Yu}^{1,2 *}$
}

Artificial modulation of electronic structures and control of the transport dynamics of carriers and excitons in CdSe nanowire are important for its application in optoelectronic nanodevices. Here, we demonstrate the aggregative flow of excitons by bending CdSe nanowires. The bending strain induces spatial variance of bandgap, and the energy bandgap gradient will result in the flow of excitons towards the bending outer edge of CdSe nanowire. The exciton emission energy shows a uniform redshift in the bending region due to the aggregative flow of excitons, and the energy redshift increases linearly with increasing the strain at the outer edge of the CdSe nanowire. Our results show an effective method to drive, concentrate, and utilize the excitons in CdSe nanowires, which provides a guide for the design of high performance and flexible optoelectronic nanodevices.

\section{INTRODUCTION}

Cadmium Selenide (CdSe) nanowire, due to its unique luminescent properties, nonlinear optical properties, quantum-size effect, and the band gap in visible light range, is a promising material for the electronic and optoelectronic nanodevices. CdSe nanowires based functional devices, such as transistors, photovoltaic cells [1], light-emitting diodes [2], lasers [3], etc., have been developed. Further modulation of the electronic structures and control of the transport dynamics of carriers in CdSe nanostructures are very important to optimize the optoelectronic properties. It has been demonstrated that the carrier and exciton transport dynamics can be effectively tuned in CdS/CdSe superlattices and core/shell nanostructures such as $\mathrm{CdTe} /$ CdSe [4] and ZnSe/CdSe [5], due to the alignment of their band structures and bandgap offset. Most recently, it has been experimentally illustrated that the colloidal core/ crown $\mathrm{CdSe} / \mathrm{CdS}$ nano-platelet based heterostructures can act as efficient exciton concentrators [6]. Besides the chemical approaches, the elastic strain can also effectively influence the electronic and optical properties of semiconductors [7-9]. The notable examples are the piezoelectricity in polar semiconductors $[10,11]$ and the strained silicon technology for enhancing carrier mobility of Si transistors [12]. The elastic strain engineering becomes even more important in semiconducting micro/nano-structures because they possess much higher mechanical toughness and strength compared to their bulk counterparts $[13,14]$. For example, significant energy redshifts of the near-band-edge (NBE) luminescence in uniaxial strain modulated $\mathrm{ZnO}$ [15] and GaAs [16] nanowires, as well as in curved $\mathrm{ZnO}$ [17-20] and CdS [21] micro/nanowires have been observed. It has also been reported that the elastic strain-gradient can effectively modulate the photoexcited carrier and exciton dynamics in $\mathrm{MoS}_{2}$ atomic membrane [7,22] and $\mathrm{ZnO}$ micro/ nanowires [23,24].

In this work, we report the efficient aggregative flow of excitons in curved CdSe nanowires. The exciton emission properties of bending CdSe nanowires with the diameter ranging from 200 to $630 \mathrm{~nm}$ have been studied by both low-temperature photoluminescence (PL) and cathodoluminescence (CL) spectra. The bending strain can induce spatial variation of bandgap and drive the exciton drift into the bending outer edge where the excitons are on the lowest energy state. The high spatial resolved CL spectra show a uniform redshift at the whole bending cross sections, which are in sharp contrast to the anti-symmetrical redshift and blueshift along the bending cross sections predicted by conventional strain theory. The uniform energy redshift increases linearly as increasing the bending curvature. Our results provide a novel method to concentrate excitons in CdSe nanowires, which will be helpful for design of high performance and flexible CdSe nanowires based optoelectronic nanodevices.

\section{RESULTS AND DISCUSSION}

The CdSe nanowires used here were synthesized via chemical vapor deposition method [25]. The CdSe nanowires have single crystal wurtzite structure and grow along the [0001] direction with diameter ranging from $200 \mathrm{~nm}$ to 1

\footnotetext{
${ }^{1}$ State Key Laboratory for Mesoscopic Physics, Department of Physics, Peking University, Beijing 100871, China

${ }^{2}$ Collaborative Innovation Center of Quantum Matter, Beijing 100871, China

${ }^{3}$ State Key Laboratory for Mechanics and Control of Mechanical Structures, and MOE Key Laboratory of Intelligent Nano Materials and Devices, Institute of Nano Science, Nanjing University of Aeronautics and Astronautics, Nanjing 210016, China

${ }^{*}$ Corresponding authors (email: liaozm@pku.edu.cn (Liao ZM); yudp@pku.edu.cn (Yu DP))
} 
$\mu \mathrm{m}$. The lattice variations of bending CdSe nanowires were studied by transmission electron microscope (TEM). To prepare the curved CdSe nanowire for TEM characterization, the CdSe nanowires were dispersed on a TEM grid with a carbon supporting film and a hole was made near the nanowire of interest by a glass tip. In a TEM (Tecnai F30), the electron beam was focused on the carbon film near the hole, and the carbon film would crimp under the irradiation of electron beam, inducing the bending of the CdSe nanowire. Then, the microstructures of the nanowire with different strain region were characterized by highresolution TEM (HRTEM) with $300 \mathrm{kV}$ electron beam. Fig. 1a shows a curved CdSe nanowire on the TEM grid. The TEM image shown in Fig. $1 \mathrm{~b}$ indicates that the bending CdSe nanowire with diameter of $\sim 200 \mathrm{~nm}$ exhibits typical inhomogeneous strain contrast in the bending region. The HRTEM images (Figs 1c and d), taken from the compressive region and the tensile region (indicated by the red dash squares in Fig. 1b), show that both inner and outer edges exhibit wurtzite crystal structure with lattice parameter $c$ of 0.69 and $0.71 \mathrm{~nm}$, respectively. The inner and outer sides are under anti-symmetric elastic compressive and tensile deformations, resulting in a linear distribution of strain in the bending cross section. The maximum bending strain can be estimated as

$$
\varepsilon= \pm \frac{\left(c_{\text {outer }}-c_{\text {inner }}\right)}{\left(c_{\text {outer }}+c_{\text {inner }}\right)}= \pm 1.34 \% .
$$

The energy band structures of CdSe crystal under elastic strain effect have been widely investigated [26-29]. It has been demonstrated that compressive and tensile strains will result in widening and narrowing bandgap in CdSe crystal, respectively. The similar conclusion has also been widely demonstrated in other semiconductors [7,15-18,21,23,24]. Therefore, an inhomogeneous strain field imposed to CdSe crystal will induce a continuous spatial variation of electronic band structures. The continuous spatial variation of energy band leads to that the excitons are drained to the low energy states. Fig. 2a schematically shows the variation of bandgap and the mechanism for aggregative flow of excitons in the bending cross section of a curved CdSe nanowire. As the strain $\varepsilon$ along the hexagonal axis [0001] in bending CdSe nanowire changes linearly from inner side (compression) to outer side (tension), both the conduction band minimum (CBM) and valence band maximum (VBM) shift linearly downwards from the inner side towards outer side, and the CBM down shifts much faster than that of the VBM. Consequently, there is a linear diminution of energy bandgap along the radial direction from inner side towards outer side in the bending CdSe nanowire (Fig. 2a). Since the thermal energy at $81 \mathrm{~K}(\sim 7 \mathrm{meV})$ is much smaller than the exciton binding energy (above $15 \mathrm{meV}$ ) of CdSe, the generated electron-hole pairs prefer to couple as excitonic states. On the other hand, although the alignments of the CBM and VBM in the banding cross section may also result in the opposite motion tendency of the electrons (towards lower $\mathrm{CB}$ ) and holes (towards higher VB), the excitons will not be decomposed into free electrons and holes by the bending strain field due to the relatively large exciton binding energy. The later PL and CL spectra of the bending CdSe nanowires also demonstrate

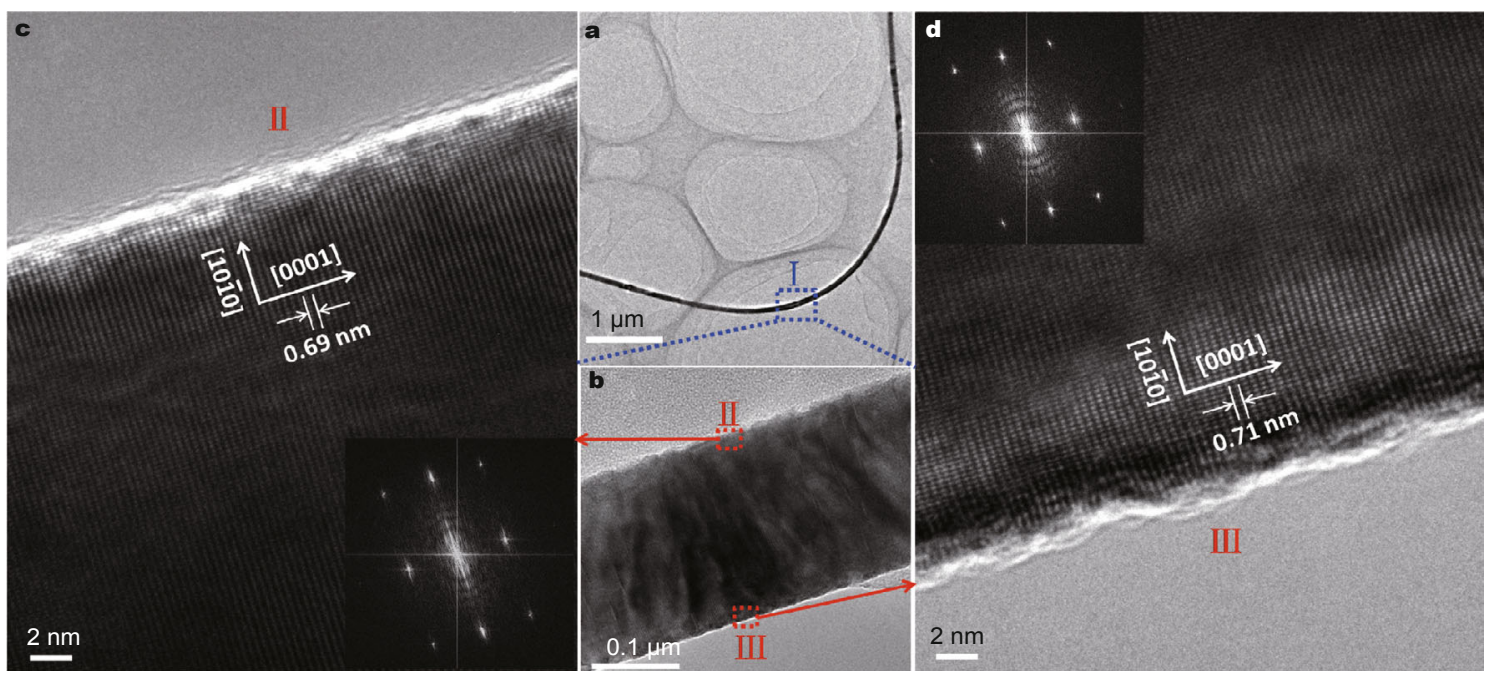

Figure 1 TEM analysis of a bending CdSe nanowire with diameter $200 \mathrm{~nm}$. (a) Low-magnification TEM image of the bending CdSe nanowire. (b) Typical strain contrasts in the area indicated by the blue square "I" in the bending region in (a). (c) and (d) High-resolution TEM images of the inner and outer edges as indicated by the red squares "II" and "III" in (b), respectively. Both inner and outer edges have wurtzite crystal structure with lattice parameter $c$ of $\sim 0.69 \mathrm{~nm}$ and $0.71 \mathrm{~nm}$, respectively. The insert figures are the fast Fourier transform patterns of the HRTEM images in (c) and (d). 

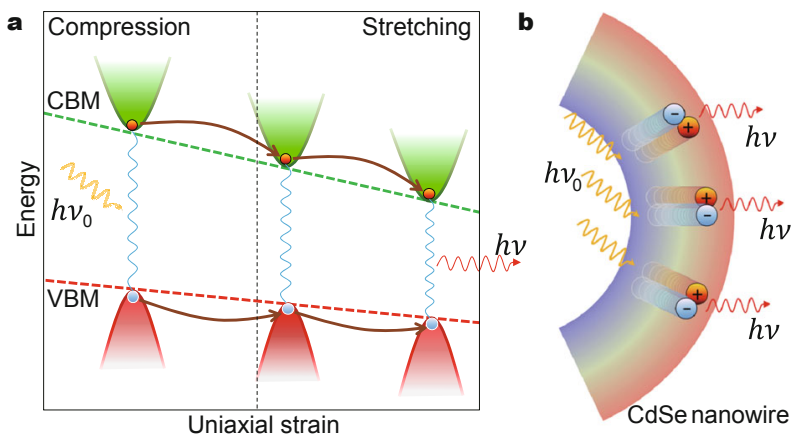

Figure 2 (a) Schematic diagram of the energy change of conduction band minimum (CBM) and valence band maximum (VBM) as a function of compressive and tensile strains in CdSe crystal and the mechanism of exciton transport dynamics. The blue wave lines indicate the strong Coulomb interactions between the electron-hole pairs, which make the electron-hole pairs keep at the excitonic states and move together under the strain gradient effect. (b) Schematic diagram of the mechanism of aggregative flow of excitons in bending CdSe nanowires. The red and blue colors represent the local tensile and compressive strains, respectively.

that the luminescence spectra are from exciton radiative recombination. The excitons of wurtzite CdSe crystal have long lifetime $(\sim 1 \mathrm{~ns}$ in bulk at $4 \mathrm{~K}[30,31]$ and $\sim 1 \mu \mathrm{s}$ in quantum dots below $10 \mathrm{~K}[32,33])$ and strong exciton diffusion effect with diffusion length of about $1 \mu \mathrm{m}$ at $10 \mathrm{~K}$ [34]. Therefore, during the lifetime before the exciton recombination, the bandgap gradient will drift the excitons generated in the bending region towards the outer edge, resulting in the aggregative flow of excitons in bending $\mathrm{CdSe}$ nanowires, as schematically shown in Fig. 2b. The dynamical equation for the excitons under strain gradient can be described as $[23,24]$

$$
\partial n(\mathbf{r}, t) / \partial t=-\mu \mathbf{F} \nabla n(\mathbf{r}, t)+D \nabla^{2} n(\mathbf{r}, t)-n(\mathbf{r}, t) / \tau,
$$

where $n(\mathbf{r}, t)$ is the density of excitons, $\mu$ is the mobility of excitons, $\mathbf{F}=\nabla \phi_{\mathrm{B}}(\mathbf{r})$ is the effective field induced by the bandgap gradient, $D$ is the diffusion coefficient of excitons, and $\tau$ is the lifetime of excitons. As most of the generated excitons can transport to the outer edge of the bending $\mathrm{CdSe}$ nanowire before recombination, the bending CdSe nanowire may act as an efficient exciton concentrator.

In order to experimentally explore the aggregative flow of excitons in bending CdSe nanowires, we carried out PL investigation on bending CdSe nanowires at low temperature $(10 \mathrm{~K})$. The as-grown CdSe nanowires were dispersed in ethanol, and then transferred onto a silicon substrate with predefined marks for identifying the nanowires of interest. Straight CdSe nanowires were formed into curved configuration by a micromanipulator under an optical microscope (Olympus, BX51M). The strong interaction between the nanowire and the substrate keeps the CdSe nanowires in curved configuration after manipulation. The curved CdSe nanowires will resile to a straight configuration when contacting a drop of ethanol, indicating that the curved CdSe nanowires are in elastic deformation. To describe the strain distribution in the curved CdSe nanowires, we define the direction which parallel to the substrate surface and perpendicular to the $c$ axis as $x$ from inside to outside, and the other one vertical to the substrate surface as $y$. In the bending region, the strain along the hexagonal axis [0001] varies linearly from compressive inside to tensile outside as $\varepsilon=x / \rho$ where $-d / 2 \leqslant x \leqslant d / 2, \rho$ is the local curvature radius, and $\mathrm{d}$ is the diameter of the nanowire. The maximum bending strains at the outer and inner edges of the curved nanowire are $\varepsilon= \pm d / 2 \rho[17,20]$, respectively.

The PL spectra were measured under UV laser (with wavelength of $325 \mathrm{~nm}$ ) excitation with the spot size of $\sim 2$ $\mu \mathrm{m}$. The temperature dependent PL spectra of a strain free CdSe nanowire $(d=600 \mathrm{~nm})$ exhibit a continuous blue shift with decreasing temperature (Fig. 3a), which is well consistent with the Varshni's law [35]. At $10 \mathrm{~K}$ only one intensive and sharp emission peak centered at $1.816 \mathrm{eV}$ with a full width at half maximum of $13 \mathrm{meV}$ is observed. Fig. $3 \mathrm{~b}$ and $\mathrm{d}$ show the typical scanning electron microscope (SEM) images of two bending CdSe nanowires on Si substrate with diameter of $400 \mathrm{~nm}$ and $500 \mathrm{~nm}$, respectively. A series of PL spectra were measured point by point along the bending CdSe nanowires at $10 \mathrm{~K}$, as indicated by the colored circles in Figs $3 \mathrm{~b}$ and $\mathrm{d}$. The corresponding $\mathrm{PL}$ spectra of these two bending CdSe nanowires are presented in Figs $3 c$ and e, respectively. At the strain free region, the PL spectrum exhibits only one sharp and intensive exciton emission peak centered at $1.816 \mathrm{eV}$. As the excitation spot gradually enters the bending region, the PL spectra display continuous redshift and then back to the original position after entering the other straight part. The experimental results are in good agreement with our theoretical analysis above. As the laser spot size is much larger than the nanowire diameter, the whole cross section of the nanowire will be covered by the laser spot, and the excitons will be generated in the whole cross section. Due to the aggregative flow of excitons in the bending CdSe nanowires, all the generated excitons in the whole cross section will transport towards the outer edge and recombine to luminescence, resulting in a redshift of the emission peak in the bending region. Besides that, the redshift of the PL spectrum increases with increasing the bending curvature, as shown in Figs $3 c$ and $e$.

To further clearly confirm the efficient aggregative flow of excitons in bending CdSe nanowires, we carefully investigated the exciton emission spectra of bending CdSe nanowires by high spatial and spectral resolution CL spectroscopy, as shown by the schematic diagram in Fig. 4a. The CL spectra were carefully collected by CL spectrom- 

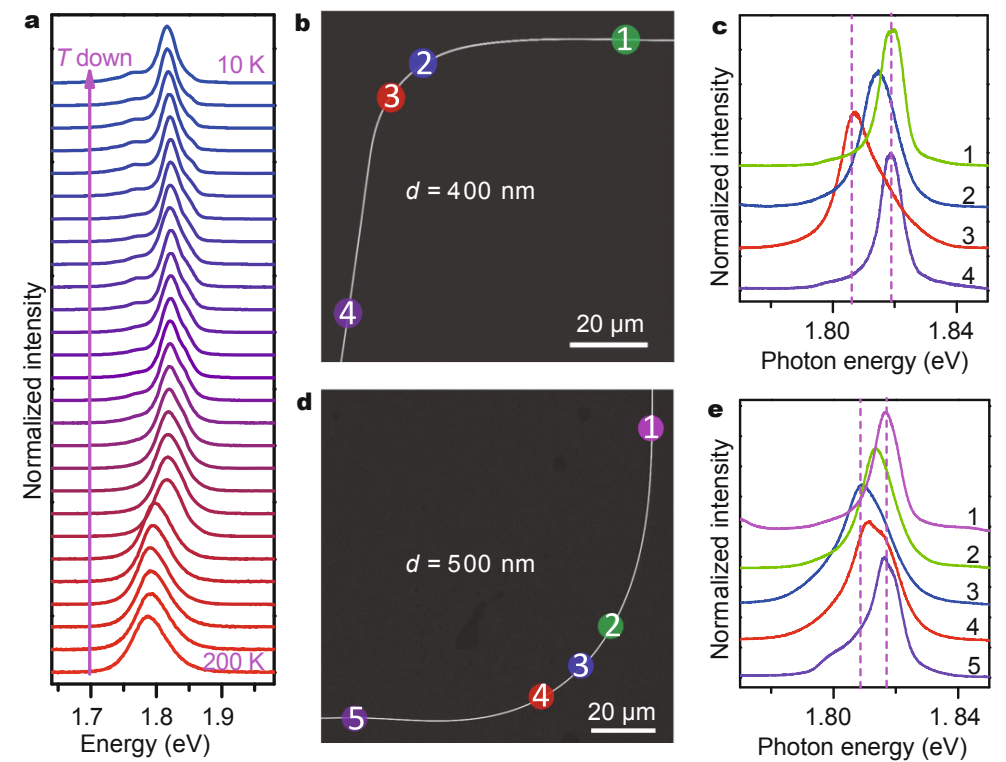

Figure 3 (a) Temperature dependent PL spectra of a strain free CdSe nanowire with diameter of 600 nm measured from $200 \mathrm{~K}$ down to $10 \mathrm{~K}$. (b) and (d) SEM images of the bending CdSe nanowires with diameter of $400 \mathrm{~nm}$ and $500 \mathrm{~nm}$, respectively. The numbered circles indicate the laser excitation positions of the PL measurements. (c) and (e) Corresponding PL spectra of the two bending nanowires shown in (b) and (d) at $10 \mathrm{~K}$, respectively. The labeled numbers of the spectrum curves corresponds to the positions marked in the SEM images. The right pink dashed line shows the initial position of exciton emission energy at the strain free region, while the left pink dashed line shows the biggest redshift position of the exciton emission energy.

eter (Gatan monocle 3+) at $\sim 6 \mathrm{~K}$ in a liquid helium flow cryostat. Fig. $4 \mathrm{~b}$ shows a typical SEM image of a bending CdSe nanowire with $d=630 \mathrm{~nm}$ on Si substrate. The CL spectra presented in Fig. $4 \mathrm{c}$ are measured via line-scanning with step size of $\sim 60 \mathrm{~nm}$ from compressive side to tensile side at the cross-sections indicated by the white arrows in the SEM image. Similar to the PL results, the CL spectra at the strain-free region exhibit only one sharp and intensive exciton emission peak. It is worth to note that the line-scanning CL spectra are identical from compressive side to tensile side at the cross-sections in the bending regions, and show a redshift and a certain degree of peak broadening compared to that from the straight part. Furthermore, the redshift increases with increasing the bending curvature. The line-scanning CL spectra at the bending cross section are in good agreement with the prediction by the aggregative flow of excitons in the bending CdSe nanowire. The excitons excited at anywhere of the bending cross section can transport to the bending outer edge and recombine to luminescence. The experimental results confirm that the bending CdSe nanowire can indeed act as an efficient exciton concentrator. To further analyze the variation of the exciton emission energy with the bending curvature, we extracted the CL spectrum from the neutral plane of each bending cross section, as indicated by the green colored spectra in Fig. 4c. The spectra from the neutral plane were then re-plotted together in Fig. 4d. It is clear that the red- shift of the exciton emission peak strongly depends on the bending curvature. The redshift increases linearly with the maximum strain $\varepsilon$ at outer edge as $\Delta E=k \cdot \varepsilon$ as shown in Fig. $4 \mathrm{e}$. The bending deformation potential $k=\partial(\Delta E) / \partial \varepsilon$ can be obtained to be $3.5 \mathrm{eV}$ through the linear fitting.

Furthermore, some other arbitrarily bending CdSe nanowires with different diameters from 200 to $600 \mathrm{~nm}$ were investigated (Fig. 5a). As shown in Fig. 5b, the CL spectra collected from the neutral plane of the bending regions demonstrate a red-shift compared to the strain-free region for all the nanowires with different diameter. All the samples show a linear relationship between the redshift of emission energy and the outermost tensile strain (Fig. 5c). Through linear fitting of the results, all the samples show the similar bending deformation potential $k=\partial(\Delta E) / \partial \varepsilon$ of $3.6 \pm 0.3 \mathrm{eV}$, which is larger than hydrostatic deformation potential $2.3 \mathrm{eV}$ [29]. This discrepancy is probably due to the different strain loading methods.

To give a more intuitive picture of the exciton aggregative flow in the bending CdSe nanowires, the CL image of the bending CdSe nanowire $(d=630 \mathrm{~nm})$ was acquired by monochromatic CL mapping mode. The SEM image of the bending region of the CdSe nanowire is shown in Fig. 6a. The monochromatic CL mapping of the bending part was obtained using $699 \mathrm{~nm}$ (the center emission wavelength of this part) for imaging is shown in Fig. 6b. The brightness of the monochromatic CL image corresponds to the collected 

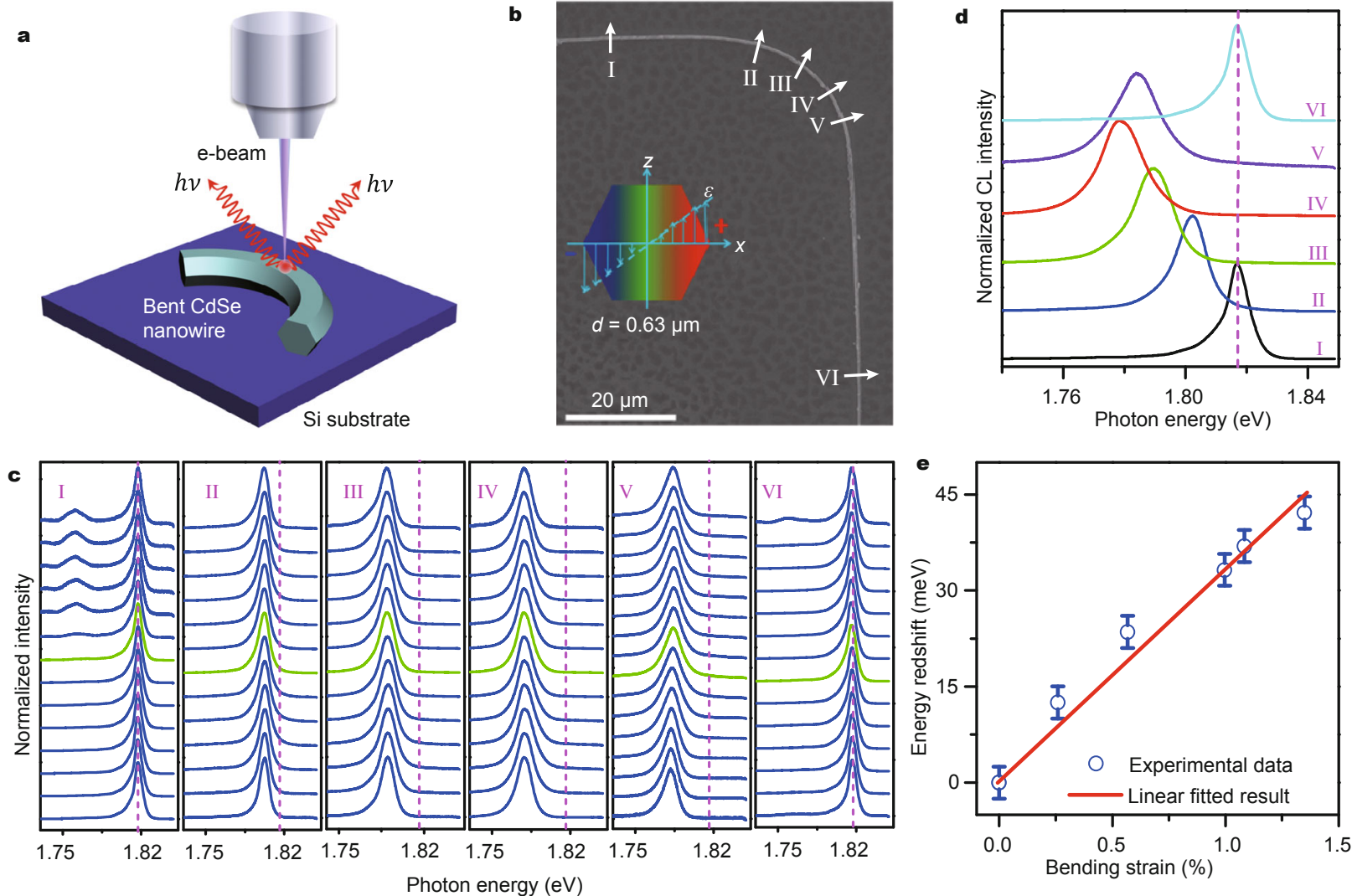

Figure $4 \mathrm{CL}$ spectra of a bending CdSe nanowire with diameter $\sim 630 \mathrm{~nm}$ at $\sim 6 \mathrm{~K}$. (a) Schematic diagram of CL measurements on the bending region of a bending CdSe nanowire. (b) SEM image of a bending CdSe nanowire. The pink arrows marked by "I"-"VI" indicate the positions, where the CL spectra were collected step-by-step from the compressive to tensile edges. (c) The line-scanning CL spectra collected at each marked positions "I"-"VI" from the inner edge to the outer edge, respectively. The pink dashed lines indicate the initial exciton emission energy position without bending strain. (d) The CL spectra extracted from the green curves in (c), which are the CL spectra measured at the neutral plane of each positions ("I"- "VI"). (e) The linear relationship between the redshift of the exciton emission energy and the outermost tensile strain at the outer edge of the nanowire. The error bars of the data are determined by the energy resolution of the CL spectroscopy.

CL intensity, i.e. the number of radiative recombined excitons. In Fig. 6b, the outer edge exhibits the highest brightness in the monochromatic CL image, which is consistent with the physical picture of the aggregative flow of exciton in bending CdSe nanowire.

\section{CONCLUSION}

In summary, via both theoretical analysis and low-temperature PL and CL investigations on the bending CdSe nanowires with different diameters (200-630 nm), we demonstrate the aggregative flow of excitons in bending CdSe nanowires. Due to the energy bandgap gradient induced by the bending strain deformation, the generated excitons in the bending region will transport towards the bending outer edge of the bending CdSe nanowires. The aggregative flow of exciton results in uniform CL spectra acquired from different positions along the bending cross section from the compressive part to the tensile part. Moreover, the exciton emission energy at the bending region has a redshift compared to the strain-free region, and the energy redshift increases linearly with increasing the outermost tensile strain. Our results pave an effective way to concentrate the excitons in CdSe nanowires, which may be valuable for the design of high performance and flexible CdSe nanowires based optoelectronic nanodevices.

\section{MATERIALS AND METHODS}

\section{Synthesis of CdSe wires}

The CdSe nanowires were synthesized via the chemical vapor deposition (CVD) method. CdSe (99.99\%) powder and Cd (99.99\%) particles were used as the growing sources, and a piece of Si wafer covered with $10 \mathrm{~nm}$ thermally evaporated Au was used as the substrate for nanowires collection. In a tube furnace, the distance between CdSe and Cd was about $2-10 \mathrm{~cm}$, and CdSe and Si substrate about 10-15 cm. Before heating, high-purity Ar gas was introduced into the quartz tube at flow rate of $100 \mathrm{sccm}$. The 

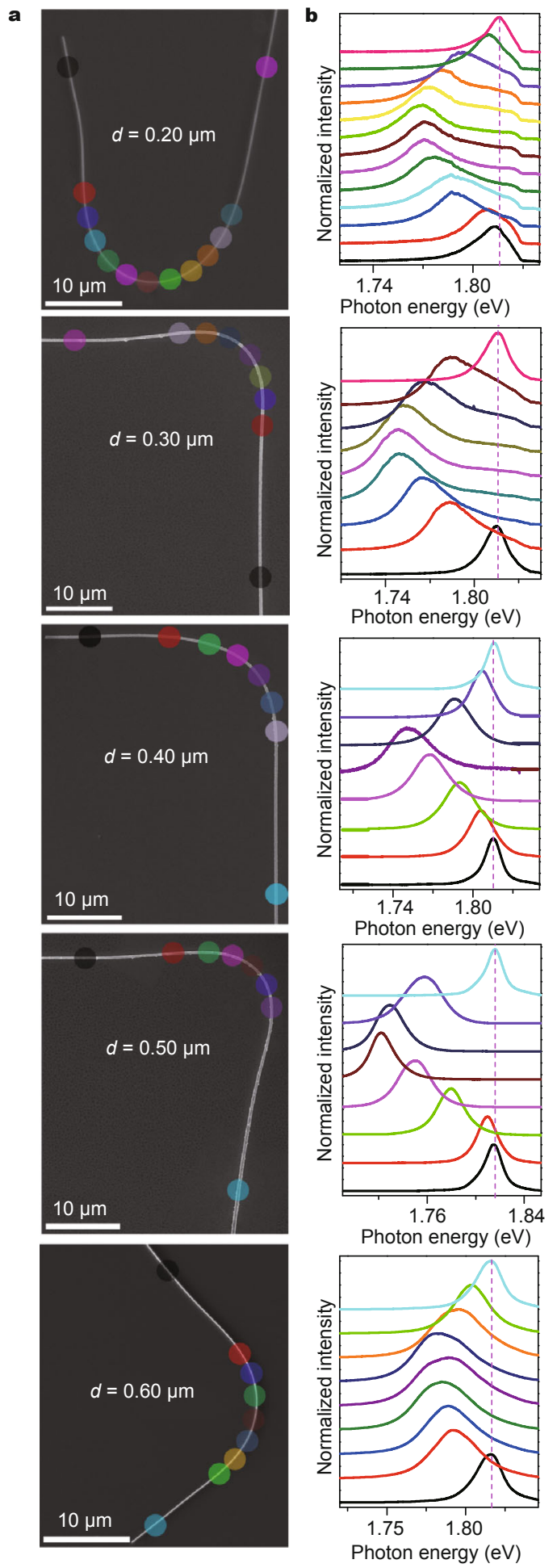
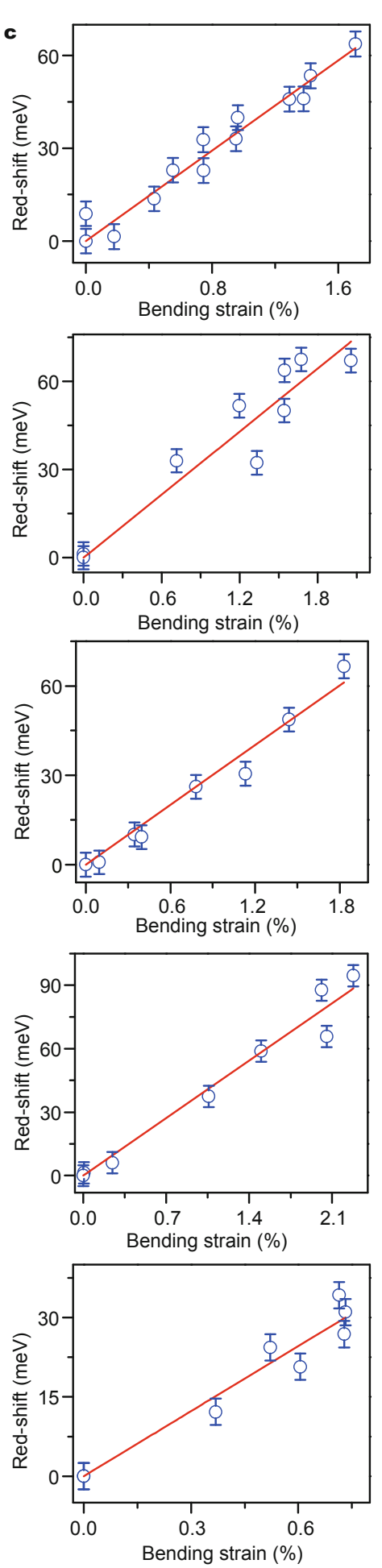

Figure 5 Bending strain effect on the exciton emission energy of bending CdSe nanowires with different diameter. (a) SEM images of the bending CdSe nanowires with diameter of 200, 300, 400, 500 and $600 \mathrm{~nm}$, respectively. The colored circles indicate the positions of CL spectra measurements. (b) The corresponding CL spectra of the bending CdSe nanowires shown in (a) at $\sim 6 \mathrm{~K}$. The spectrum curves measured from the neutral plane of the bending cross section at the positions with colored circles in the SEM images were presented using the same color. The pink dashed lines show the initial position of exciton emission energy without bending strain. (c) The linear relationship between the redshift of the exciton emission energy and the maximum bending strain at the outer edge of the nanowire. 

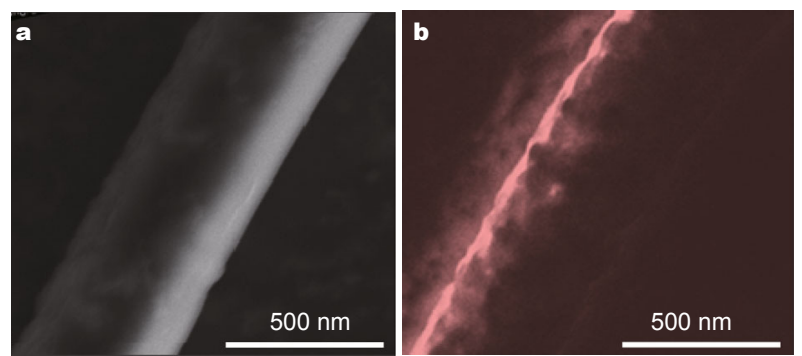

Figure 6 (a) SEM image of a bending CdSe nanowire $(d=630 \mathrm{~nm})$. (b) The corresponding monochromatic CL mapping image at wavelength of $699 \mathrm{~nm}$.

quartz tube was pumped for 20 min to clear the oxygen, and then the furnace was rapidly heated to $700^{\circ} \mathrm{C}$. During growth, the high-purity Ar flow rate was kept at $100 \mathrm{sccm}$. The local temperatures for $\mathrm{CdSe}, \mathrm{Cd}$, and $\mathrm{Si}$ substrate were about $700^{\circ} \mathrm{C}, 600-700^{\circ} \mathrm{C}$, and $500-600^{\circ} \mathrm{C}$, respectively. The synthesis duration was about $0.5 \mathrm{~h}$.

\section{CL measurements}

To obtain the optimum spatial resolution with best signalto-noise ratio, an electron beam was accelerated at $10 \mathrm{kV}$ (spot size 4), which resulted in the effective interaction of electron beam in CdSe of about $100 \mathrm{~nm}$ (with $90 \%$ power in this region, as supported by Monte Carlo simulation [36]). The CL spectra were carefully collected by CL spectroscopy (Gatan monocle 3+) at liquid helium temperature. In the line-scanning mode, the CL spectra were collected step by step along the radial direction across the diameter of the CdSe nanowires from inner side to outer side, with step size of ca. $60 \mathrm{~nm}$. In the spot-scanning mode, the CL spectra were collected at the center of the radial cross-sections. The CL spectra were recorded by CCD (Charge Coupled Device) in a scanning range of 300 to $450 \mathrm{~nm}$ at spectral resolution of about $0.5 \mathrm{~nm}$.

\section{Received 12 October 2014; accepted 30 October 2014;} published online 8 December 2014

1 Gur I, Fromer NA, Geier ML, Alivisatos AP. Air-stable all-inorganic nanocrystal solar cells processed from solution. Science, 2005, 310: 462-465

2 Achermann M, Petruska MA, Kos S, et al. Energy-transfer pumping of semiconductor nanocrystals using an epitaxial quantum well. Nature, 2004, 429: 642-646

3 Ma C, Ding Y, Moore D, Wang X, Wang ZL. Single-crystal CdSe nanosaws. J Am Chem Soc, 2004, 126: 708-709

4 Smith AM, Mohs AM, Nie S. Tuning the optical and electronic properties of colloidal nanocrystals by lattice strain. Nat Nanotech, 2008, 4: 56-63

5 Flack F, Samarth N, Nikitin V, et al. Near-field optical spectroscopy of localized excitons in strained CdSe quantum dots. Phys Rev B, 1996, 54: R17312

6 Tessier MD, Spinicelli P, Dupont D, et al. Efficient exciton concentrators built from colloidal core/crown CdSe/CdS semiconductor nanoplatelets. Nano Lett, 2013, 14: 207-213

7 Feng J, Qian X, Huang CW, Li J. Strain-engineered artificial atom as a broad-spectrum solar energy funnel. Nat Photonics, 2012, 6: 866-872

8 Guinea F, Katsnelson M, Geim A. Energy gaps and a zero-field quantum Hall effect in graphene by strain engineering. Nat Phys, 2009, 6: 30-33

9 Fu XW, Liao ZM, Liu R, Xu J, Yu DP. Size-dependent correlations between strain and phonon frequency in individual $\mathrm{ZnO}$ nanowires. ACS Nano, 2013, 7: 8891-8898

10 Wang ZL, Song J. Piezoelectric nanogenerators based on zinc oxide nanowire arrays. Science, 2006, 312: 242-246

11 Dong L, Niu S, Pan C, et al. Piezo-phototronic effect of CdSe nanowires. Adv Mater, 2012, 24: 5470-5475

12 Ieong M, Doris B, Kedzierski J, Rim K, Yang M. Silicon device scaling to the sub-10-nm regime. Science, 2004, 306: 2057-2060

13 Zhu T, Li J. Ultra-strength materials. Prog Mater Sci, 2010, 55: 710-757

14 Wang L, Zheng K, Zhang Z, Han X. Direct atomic-scale imaging about the mechanisms of ultralarge bent straining in Si nanowires. Nano Lett, 2011, 11: 2382-2385

15 Wei B, Zheng K, Ji Y, et al. Size-dependent bandgap modulation of ZnO nanowires by tensile strain. Nano Lett, 2012, 12, 4595-4599

16 Signorello G, Karg S, Bjork MT, Gotsmann B, Riel H. Tuning the light emission from GaAs nanowires over $290 \mathrm{meV}$ with uniaxial strain. Nano Lett, 2013, 13: 917-924

17 Han X, Kou L, Lang X, et al. Electronic and mechanical coupling in bent $\mathrm{ZnO}$ nanowires. Adv Mater, 2009, 21: 4937-4941

18 HanX, Kou L, Zhang Z, et al. Strain-gradient effect on energy bands in bent $\mathrm{ZnO}$ microwires. Adv Mater, 2012, 24: 4707-4711

19 Xu S, Guo W, Du S, Loy M, Wang N. Piezotronic effects on the optical properties of ZnO nanowires. Nano Lett, 2012, 12: 5802-5807

20 Liao ZM, Wu HC, Fu Q, et al. Strain induced exciton fine-structure splitting and shift in bent $\mathrm{ZnO}$ microwires. Sci Rep, 2012, 2: 452

21 Sun L, Kim DH, Oh KH, Agarwal R. Strain-induced large exciton energy shifts in buckled CdS nanowires. Nano Lett, 2013, 13: 3836-3842

22 Castellanos-Gomez A, Roldán R, Cappelluti E, et al. Local strain engineering in atomically thin MoS2. Nano Lett, 2013, 13: 5361-5366

23 Fu X, Su C, Fu Q, et al. Tailoring exciton dynamics by elastic straingradient in semiconductors. Adv Mater, 2014, 26: 2572-2579

24 Fu X, Jacopin G, Shahmohammadi M, et al. Exciton drift in semiconductors under uniform strain gradients: application to bent ZnO microwires. ACS Nano, 2014, 8: 3412-3420

25 Liu C, Wu P, Sun T, et al. Synthesis of high quality n-type CdSe nanobelts and their applications in nanodevices. J Phys Chem C, 2009, 113: 14478-14481

26 Yang S, Prendergast D, Neaton JB. Nonlinear variations in the electronic structure of II-VI and III-V wurtzite semiconductors with biaxial strain. Appl Phys Lett, 2011, 98: 152108

27 Choi CL, Koski KJ, Sivasankar S, Alivisatos AP. Strain-dependent photoluminescence behavior of CdSe/CdS nanocrystals with spherical, linear, and branched topologies. Nano Lett, 2009, 9: 3544-3549

28 Yang S, Prendergast D, Neaton JB. Strain-induced band gap modification in coherent core/shell nanostructures. Nano Lett, 2010, 10: 3156-3162

29 Shan W, Walukiewicz W, Ager III J, et al. Pressure dependence of the fundamental band-gap energy of CdSe. Appl Phys Lett, 2004, 84: 67-69

30 Bawendi MG, Carroll P, Wilson WL, Brus L. Luminescence properties of CdSe quantum crystallites: resonance between interior and surface localized states. J Chem Phys, 1992, 96: 946-954

31 Nirmal M, Murray C, Bawendi M. Fluorescence-line narrowing in CdSe quantum dots: surface localization of the photogenerated exciton. Phys Rev B, 1994, 50: 2293-2300 
32 de Mello Donegá C, Bode M, Meijerink A. Size and temperature-dependence of exciton lifetimes in CdSe quantum dots. Phys Rev B, 2006, 74: 085320

33 Crooker S, Barrick T, Hollingsworth J, Klimov V. Multiple temperature regimes of radiative decay in CdSe nanocrystal quantum dots: intrinsic limits to the dark-exciton lifetime. Appl Phys Lett, 2003 82: 2793-2795

34 Erland J, Razbirin B, Pantke KH, Lyssenko V, Hvam J. Exciton diffusion in CdSe. Phys Rev B, 1993, 47: 3582-3587

35 Varshni Y. Temperature dependence of the energy gap in semiconductors. Physica, 1967, 34: 149-154

$36 \mathrm{Xu}$ J, Chen L, Yu L, et al. Cathodoluminescence study of InGaN/ GaN quantum-well LED structures grown on a Si substrate. J Electron Mater, 2007, 36: 1144-1148
Acknowledgements This work was supported by the Ministry of Science and Technology of China (2013CB932602, 2013CB934600), the National Natural Science Foundation of China (NSFC) (11274014, 11234001), and the Program for New Century Excellent Talents in University of China (NCET-12-0002).

Author contributions Yu D initiated and guided the project. Fu X and Liao ZM designed the experiments. Fu X, Zhu R and Xu J performed the experiments. Ye Y and Dai L synthesized the CdSe nanowires. Fu X and Liao ZM analyzed the data and wrote the paper. All authors contributed to discussion of the results and reviewed the manuscript.

Conflict of interest The authors declare no competing financial interest.

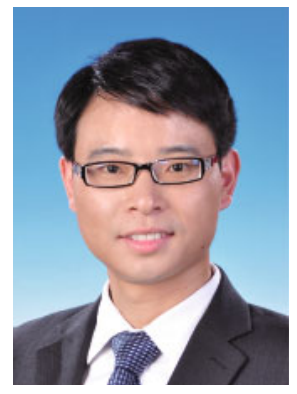

Xuewen Fu received his BSc degree (2009) in physics from Beijing Normal University and PhD degree in physics from Peking University in 2014. He spent half a year working as an exchange scholar at the Quantum Optoelectronic Laboratory, École Polytechnique Fédérale de Lausanne (EPFL). His current research interests include electronic-mechanical coupling and photoelectronics of micro/nano-semiconductors, as well as ultrafast photocarrier (exciton, electron and hole) transport and structural dynamics in novel nanostructures and applications.

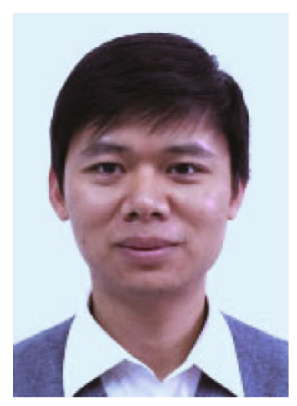

Zhi-Min Liao received his BSc degree from Sun Yat-sen University in 2002 and PhD degree from Peking University in 2007. Thereafter, he joined the School of Physics at Peking University as a Lecturer and then became an associate professor in 2011. Meanwhile he spent one year working as a postdoctoral fellow in Trinity College Dublin. His current research is focused on quantum transport in semiconductor nanowires, graphene, topological insulators, and atomically layered materials.

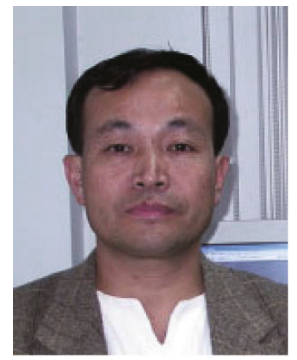

Dapeng YU is a professor of School of Physics, Peking University. He received his BSc from East-China University, Shanghai, MSc at the Shanghai Institute of Ceramics, Chinese Academy of Sciences, and PhD from Laboratire de Physique des Solides, Université Paris-sud, Orsay, France. His research interests include developing controllable methods to fabricate semiconductor nanowires, exploring their properties and tunability, and applications in novel energy; quantum transport in graphene and other 2D materials; nano-electromechanical devices and sensing; solid-state nanopore sequencing.

中文摘要 调制CdSe纳米线的电子结构和发光性质对于进一步开发其在光电子功能器件中的应用具有十分重要的意义. 本研究揭示了弯 曲 CdSe纳米线中激子流的汇聚效应. 弯曲形变在CdSe纳米线中形成从弯曲内侧到弯曲外侧连续变化的带隙, 从而激子在能量梯度诱发的 等效内建场的驱动下向较低能量的弯曲外侧漂移和汇聚. 由于激子迁移到弯曲外侧边缘并复合发光, 使得激子发光光谱在弯曲横截面发 生整体红移, 并且发光能量的红移量随弯曲外侧边缘的最大拉伸应变线性增加. 本文展示了一种新的驱动、汇聚乃至利用 CdSe纳米线激 子的有效方法, 对于开发和设计高性能的柔性光电子器件具有重要的指导意义. 